SU-ITP-95-4

hep-th/9503029

March 5, 1995

\title{
BLACK HOLE MULTIPLETS AND SPONTANEOUS BREAKING OF LOCAL SUPERSYMMETRY
}

\author{
Renata Kallosh 円 \\ Physics Department, Stanford University, Stanford CA 94305
}

\begin{abstract}
We classify states saturating a double or a single supersymmetric positivity bound of a four-dimensional $N=4$ supersymmetry. The massive four-dimensional doublebound states (Bogomolny states) are shown to form a light-like representation of tendimensional supersymmetry. The single-bound states form a massive representation (centrino multiplet) of a four-dimensional supersymmetry. The first component of the centrino multiplet is identified with extreme black holes with regular horizon which have one quarter of unbroken supersymmetry. The centrino multiplet includes a massive spin 3/2 state, the centrino, as a highest spin state.

Existence of massive black hole supermultiplets may affect the massless sector of the theory. Assuming that gluino condensate is formed one can study its properties. The bilinear combination of covariantly constant Killing spinors supplies the possible form for a gluino condensate. The condensate has null properties, does not introduce a cosmological constant, and may lead to a spontaneous breaking of local supersymmetry. This suggests that centrino may provide a consistent super-Higgs mechanism.
\end{abstract}

\footnotetext{
${ }^{1}$ E-mail: kallosh@physics.stanford.edu
} 


\section{Introduction}

In this paper we are going to present several different results concerning physics of extreme black holes in supersymmetric theories. We will begin with the problem of classification of extreme black holes. This classification depends crucially on the properties of black holes with respect to supersymmetry transformations. According to [1], some of the black holes are supersymmetric under one quarter of supersymmetries of the original theory. We will show that they and their superpartners form a massive supermultiplet of highest spin 3/2. Some other black holes are symmetric with respect to one half of original supersymmetries. They form a massive supermultiplet of spin 1. Such black holes form a massive stringy excitations of the four-dimensional theory. We will show that in fact they form a light-like representation from the point of view of the underlying $d=10$ string theory.

Massive supermultiplets of highest spin 3/2 have been studied many years ago, but then discarded as having no realistic implementations in particle physics, see, e.g., [2]. As we will see, these multiplets should be reinstated in their rights if in addition to particles appearing as elementary field excitations one also considers extreme black holes and their superpartners. At this level one can obtain a new example of a consistent theory of massive particles (solitons) with spin $3 / 2$, which we called centrino. It would be interesting to consider a possibility of mixing of centrino and gravitino, which would make gravitino massive.

Extreme black holes have many different "faces". They can be considered as point-like objects in $d=4$. However, being uplifted to $d=10$, some of them look like gravitational waves, some may be brought to the form of gravitational self-dual instantons. Then one can again dimensionally reduce these solutions to $d=4$. This can be done in different ways, using duality symmetries and also making different choices for a four-dimensional manifold. Some of these ways produce black holes, in some other ways one can obtain specific gravitational instantons. This reminds the relation between static monopoles in four-dimensional Minkowski space $\left(t, x^{1}, x^{2}, x^{3}\right)$ and self-dual instantons in the four-dimensional Euclidean space $\left(x^{1}, x^{2}, x^{3}, x^{4}\right)$ in Yang-Mills theory.

When analysing these configuration we have found that all of them possess a very interesting property, related to the fact that they admit supercovariantly constant Killing spinors.

One may try to solve equation of motion for the gluino field in the presence of extreme black holes. It so happens that the solution for the gluino field can be easily obtained, and that it does not influence in any way the original black hole solutions. Thus, extreme black hole solutions admit existence of a gluino field. This gluino field is analogous in a certain sense to the gluino condensate introduced in [3] in an attempt to obtain a mechanism of supersymmetry breaking in string theory. However, unlike the gluino condensate proposed in [3], our gluino field, even without the 3 -form condensate, does not introduce any cosmological constant. This invites us to look very attentively at the black hole physics from the point of view of stringy phenomenology and spontaneous breaking of local supersymmetry. 


\section{$2 \quad$ SUSY bounds}

The ultimate importance of supersymmetric positivity bounds has been realized by Witten and Olive [4] in supersymmetric Yang-Mills theories and by Gibbons and Hull [5] in gravitational theories. After the black hole solutions saturating the Bogomolny bound of $N=2$ supersymmetry have been found [5] there was an extensive study of other solutions of gravitational theories, which have some kind of unbroken supersymmetries. The purpose of this section is to classify supermultiplets satisfying either a single or a double supersymmetric bound of $N=4$ supersymmetry. This classification will give some support to the possibility of having a massless graviton and a massive gravitino in a four-dimensional theory, without explicit breaking of supersymmetry and without introducing the cosmological constant.

In what follows we would like to classify the known black hole type configurations according to the supersymmetry bound (bounds) which they saturate. Extended supersymmetries have various representations with specific properties related to the supersymmetry algebra. An extensive review of the representations of extended supersymmetry algebra in various dimensions was given by Strathdee [2]. The supersymmetry algebra defines some special representations whose existence is connected with the saturation of supersymmetric positivity bounds. Some recent studies of the monopoles, extreme black holes, gravitational waves and string states were associated with such bounds.

In global $N$-extended supersymmetric theories $\frac{N}{2}\left(\frac{N-1}{2}\right.$ for odd $\left.N\right)$ supersymmetric positivity bounds arise as an intrinsic property of the supersymmetry algebra with central charges. The bounds were explicitly derived by Ferrara, Savoy and Zumino [6] starting from the following algebra:[7]

$$
\left\{Q_{\alpha}^{i}, Q_{\beta}^{j}\right\}=\left(\gamma^{\mu} C\right)_{\alpha \beta} P_{\mu} \delta^{i j}+C_{\alpha \beta} U^{i j}+\left(\gamma_{5} C\right)_{\alpha \beta} V^{i j}, \quad i, j=1, \ldots N, \quad \alpha=1, \ldots 4 .
$$

In the Majorana representation $Q_{\alpha}^{i}$ are hermitian. The operators $U$ and $V$ are hermitian and belong to the centre of the superalgebra. In Weyl basis in two-component notations the anticommutators become

$$
\begin{aligned}
\left\{Q_{\alpha}^{i}, Q_{\dot{\beta}}^{j *}\right\} & =\left(\sigma^{\mu} C\right)_{\alpha \dot{\beta}} P_{\mu} \delta^{i j} \\
\left\{Q_{\alpha}^{i}, Q_{\beta}^{j}\right\} & =\epsilon_{\alpha \beta} Z^{i j} \\
\left\{Q_{\dot{\alpha}}^{i *}, Q_{\dot{\beta}}^{j *}\right\} & =\epsilon_{\dot{\alpha} \dot{\beta}} Z^{* i j}
\end{aligned}
$$

The matrix $Z^{i j} \equiv-V^{i j}+i U^{i j}$ is complex skew symmetric. In what follows we will restrict ourself to the $N=4$ case. In Weyl basis the $U(4)$ transformation of supersymmetry charges

$$
Q_{\alpha}^{i} \rightarrow \mathcal{U}^{i j} Q_{\alpha}^{j}=\tilde{Q}_{\alpha}^{i}, \quad Q_{\alpha}^{* i} \rightarrow \mathcal{U}^{* i j} Q_{\alpha}^{* j}=\tilde{Q}_{\alpha}^{*} i
$$

leaves eq. (2) unchanged and forces the central charges to transform like the complex antisymmetric $[4]_{2}$ representation of $U(4)$,

$$
Z^{i j} \rightarrow\left(\mathcal{U} Z \mathcal{U}^{T}\right)^{i j}=\tilde{Z}^{i j}
$$

\footnotetext{
2 The notation here are as in [6].
} 
Using this $U(4)$ freedom of a choice of a basis for the supersymmetry charges one can bring the complex antisymmetric matrix to a normal form?

$$
\tilde{Z}=i \sigma_{2} \times\left(\begin{array}{cc}
z_{1} & 0 \\
0 & z_{2}
\end{array}\right)
$$

where $\sigma_{2}$ is the Pauli matrix and $\hat{Z}$ is a $2 \times 2$ diagonal matrix with non-negative real eigenvalues $z_{1}, z_{2}$. After some additional symplectic rotation of spinorial charges the supersymmetry algebra in the rest frame acquires the following form:

$$
\begin{aligned}
& \left\{S_{(1)}, S_{(1)}^{*}\right\}=2\left|S_{(1)}\right|^{2}=m-z_{1} \geq 0, \\
& \left\{S_{(2)}, S_{(2)}^{*}\right\}=2\left|S_{(2)}\right|^{2}=m-z_{2} \geq 0, \\
& \left\{T_{(1)}, T_{(1)}^{*}\right\}=2\left|T_{(1)}\right|^{2}=m+z_{1} \geq 0, \\
& \left\{T_{(2)}, T_{(2)}^{*}\right\}=2\left|T_{(2)}\right|^{2}=m+z_{2} \geq 0 .
\end{aligned}
$$

The invariances of the algebra (2) with non-vanishing central charges are classified by the orbits of the twofold antisymmetric representation of $[N]_{2}$ of $U(4)$. The generic orbit has a little group $(U S p(2))^{2}$. Thus in general, for arbitrary central charges the supersymmetry algebra (2) is invariant under $(U S p(2))^{2}$ symmetry. In special cases when $z_{1}>z_{2}$ and $z_{2}=0$ the critical orbit has a little group $U S p(2) \times U(2)$. The case $z_{1}=z_{2}$ corresponds to the special critical orbit with $U S p(4)$ invariance.

The two positivity bounds on states in $N=4$ supersymmetry

$$
m \geq z_{1}, \quad m \geq z_{2},
$$

exist because these combinations of the mass and central charges of all states can be expressed through the square of a particular supersymmetry generators acting on that state. The structure of multiplets and its dimension depend crucially on whether one or both supersymmetric positivity bounds are saturated. Saturation of one bound inactivates one fourth of the components of the supersymmetry charge, leaving the remaining three quarters to generate all states of the multiplet, starting with the zero helicity state. Saturation of the two bounds leaves only one half of supersymmetry generators to create the states of the multiplet. Thus there is a strong discontinuity in the dimension of the supermultiplet between the state saturating only one bound which may even be very close to saturating two bounds and the state which actually saturate both bounds. This reminds us of the situation in the Yang-Mills theory: small mass still means 3 polarization states, whereas the massless state has only 2.

\footnotetext{
${ }^{3}$ It is always possible for one irreducible multiplet, it may not be possible for few multiplets simultaneously.

${ }^{4}$ There is one more set of operators with the same algebra.
} 


\section{Centrino multiplet saturating the single bound of $\quad N=$ 4 SUSY}

Consider the states saturating the single bound $m=z_{1}$ with $z_{1}>z_{2}$. It is obvious from eq. (6) that saturated bound $m=z_{1}$ with $z_{1}>z_{2}$ means that the generators $S_{(1)}$ drop from the spectrum generating Clifford algebra. Instead of 16 supersymmetry charges we are left with 12 . For one half of them we get

$$
\begin{aligned}
& \left\{S_{(2)}, S_{(2)}^{*}\right\}=2\left|S_{(2)}\right|^{2}=z_{1}-z_{2}, \\
& \left\{T_{(1)}, T_{(1)}^{*}\right\}=2\left|T_{(1)}\right|^{2}=2 m, \\
& \left\{T_{(2)}, T_{(2)}^{*}\right\}=2\left|T_{(2)}\right|^{2}=z_{1}+z_{2} .
\end{aligned}
$$

The second set of generators has the same structure.

After the rescaling under the strict condition of splitting of the central charges $z_{1} \neq z_{2}$ we get

$$
\begin{aligned}
Q_{1} & \equiv\left(\frac{m}{z_{1}-z_{2}}\right)^{\frac{1}{2}} S_{2}, \\
Q_{2} & \equiv \sqrt{2} T_{1}, \\
Q_{3} & \equiv\left(\frac{m}{z_{1}+z_{2}}\right)^{\frac{1}{2}} T_{2} .
\end{aligned}
$$

The rescaled generators $Q^{i}, i=1,2,3$, form the algebra

$$
\left\{Q_{i}, Q_{j}^{*}\right\}=\delta_{i j} m
$$

(together with the second set) defining the massive representation without central charges of $N=3$ supersymmetry repeated twice. The spectrum generating Clifford algebra can be brought to the $S O(12)$ invariant form with the states classified by $S U(2) \times U S p(6)$. The $S U(2)$ spin will run inside the fundamental multiplet from $J=0$ up to $J=\frac{3}{2}$. We will call the 32 -dimensional multiplet with such properties a centrino multiplet. The zero helicity state will be called a holon to reflect the fact that $U(1)^{2}$ black holes, including Reissner-Nordström dyons with one quarter of unbroken $N=4$ supersymmetry provide the zero helicity state for this multiplet [1]. The spin one-half state will be named holino?. The next partner, spin one state will be called a centron,

\footnotetext{
${ }^{5}$ If we would consider only the Reissner-Nordström black holes embedded into $N=2$ supersymmetric theory with one central charge and one half of supersymmetries unbroken, the corresponding multiplet [5] would have the highest $S U(2)$ spin $\frac{1}{2}$ and would consists of the holon and holino and could be called a holino multiplet. The analogous soliton of global $N=2$ supersymmetry which has a monopole as the zero helicity state has a spin one-half partner, sometimes called monopolino and the total multiplet may be called a monopolino multiplet in accordance with the name of the highest spin state.
} 
to reflect the fact that this massive representation of supersymmetry carries a central charge. Finally, the highest spin $\frac{3}{2}$ state, centrino will provide the name of the total multiplet.

It is quite remarkable that Strathdee [2] gave a detailed description of this multiplet and of the quantum numbers of supersymmetry charges and central charges according to the rest symmetry group $S O(3) \times S U(2) \times S U(2) \times U(1)$. He also explained that this multiplet contains massive spin $\frac{3}{2}$ states. Therefore he suggested "to discard this possibility" and presented his work in the abstract as a "catalogue of all supermultiplets whose states carry helicity $\leq 2$ in the massless cases and $\leq 1$ in the massive case". Simultaneously in the middle of his work he has a very important observation that "It is not altogether clear what should constitute an acceptable massive representation but we shall restrict our considerations to those which include spins $\leq 1$. This reflects the current situation in local field theory - at least insofar as theories with a finite number of field multiplets are concerned ...". On the other hand, it was anticipated in the early days of supersymmetry by Fayet and Sohnius as well as by Ferrara, Savoy and Zumino [6] (who described this multiplet in all details) that such multiplets might play a crucial role for a super-Higgs effect.

The new attitude to this representation which we are strongly promoting here came out from the more recent study of extreme black holes, which gave a non-trivial example of such multiplet. One may even ignore black holes and simply reinstate the massive spin $\frac{3}{2}$ multiplet. One may try to look for this multiplet directly in particle physics by searching for a composite-field multiplet. The most interesting massive multiplets with central charges which are represented by the black hole solutions have the highest spin state given by

$$
J_{\max }=\frac{N-q}{2}
$$

where $N$ is the number of supersymmetries, $q \leq \frac{N}{2}\left(q \leq \frac{N-1}{2}\right.$ for odd $\left.N\right)$ is the number of bounds, which are saturated. Thus for $N=2$ we have only one bound available and therefore $J_{\max }=\frac{2-1}{2}=\frac{1}{2}$. For $N=4$ we can saturate either two bounds and have $J_{\max }=\frac{4-2}{2}=1$ or one bound and have $J_{\max }=\frac{4-1}{2}=\frac{3}{2}$. The known $N=2$ example includes the Reissner-Nordström black hole embedded into $\stackrel{2}{N}=2$ supergravity with $J_{\max }=\frac{2-1}{2}=\frac{1}{2}$, holino. The second type with $J_{\max }=1$ includes all states saturating a double supersymmetric bound (Bogomolny bound) of $N=4$ supergravity, in particular pure electric and pure magnetic dilaton black holes and axiondilaton black holes. They form a centron multiplet. All string states in Sen-Schwarz [7] spectrum also form centron multiplets. The relation between string states and extreme black holes was studied in [8]. Finally, $U(1)^{2}$ black holes give an example of $J_{\max }=\frac{3}{2}$, centrino multiplet.

It seems plausible that when $N=4$ supersymmetry is involved, which typically has only massless multiplets including gravitons, gluons and photons in field theory, the source of the mass parameter can come only from some solitons carrying the ADM mass of a configuration. This is exactly why before extreme black holes where embedded into $N=4$ supersymmetry with one

\footnotetext{
${ }^{6}$ For $N=3$ there exists a massive multiplet (without central charges, $q=0$ ) with $J_{\max }=\frac{3}{2}$ and a state saturating one bound with $J_{\max }=\frac{3-1}{2}=1$. However, the states of $N=3$ supersymmetry have not been identified yet with known solitons.
} 
quarter of unbroken supersymmetries there was no clear or constructive reason to bring massive spin $\frac{3}{2}$ multiplets into the search of a mechanism of spontaneous breaking of local supersymmetry.

\section{Centron multiplet saturating the double bound of $N=$ 4 SUSY}

If the strong bound $m=z_{1}=z_{2}$ is saturated the charges $S_{(2)}$ as well as $S_{(1)}$ drop from the spectrum generating Clifford algebra. We are left with $S O(8)$ invariant Clifford algebra with the states classified by $S U(2) \times U S p(4)$. The one-particle states are those of massive representation of $d=4, N=2$ supersymmetry algebra without central charges repeated twice. The $S U(2)$ spin will run from 0 to 1 .

The algebra of $N=4$ extended supersymmetries without central charges has $U(4)$ symmetry. The existence of 12 central charges $Z^{i j}$ reduces this symmetry to the subgroups of $U(4)$ which leave the complex skew symmetric numerical $4 \times 4$ matrix $Z^{i j}$ invariant. First of all we would like to stress that not all representations of four-dimensional $N=4$ supersymmetry algebra with central charges can be identified with some representation of ten-dimensional supersymmetry algebra [6], but only the representations with exactly equal $z_{1}=z_{2}$.

Even more striking is the fact that the four-dimensional massive representation saturating the double bound of $N=4$ SUSY can be identified with the light-like representation of the ten-dimensional global supersymmetry algebra [6]. Light-like representation are the massless representation of supersymmetry without central charges. In what follows, we will explain that the Bogomolny bound can be reexpressed as the statement that all states in ten-dimensional supersymmetric theories have a non-negative mass,

$$
m_{4} \geq z_{1}=z_{2} \quad \Longleftrightarrow \quad m_{10} \geq 0 .
$$

Saturation of the double bound in ten-dimensional language is equivalent to light-likeness of the representation,

$$
m_{4}=z_{1}=z_{2} \quad \Longleftrightarrow \quad m_{10}=0 .
$$

In view of the fact that the states saturating the Bogomolny bound have been under investigation recently, it might be useful to present the explanation of the relation above. Indeed, it is known in principle that $N=4, d=4$ supersymmetry comes from $N=1, d=10$ supersymmetry. However, only the representations of four-dimensional supersymmetry with the central charges coinciding do correspond to the global representation of $d=10$ SUSY. The argument goes as follows [6]. The $N=1$ ten-dimensional SUSY algebra is

$$
\left\{Q_{\alpha}, Q_{\beta}\right\}=-\left(\gamma^{\mu} C\right)_{\alpha \beta} P_{\mu}-\left(\gamma^{A} C\right)_{\alpha \beta} P_{A}, \quad \alpha=1, \ldots 16, \quad A=1, \ldots 6,
$$

where the SUSY charge is a Majorana-Weyl ten-dimensional spinor. The question is: under which conditions $\left(\gamma^{A} C\right)_{\alpha \beta} P_{A}$ in some basis can be brought to the four-dimensional form $\left(C_{4}\right)_{\alpha \beta} U^{i j}+$ 
$\left(\gamma_{5}\left(C_{4}\right)\right)_{\alpha \beta} V^{i j}$ so that the ten-dimensional $N=1$ algebra coincides with the four-dimensional $N=4$ SUSY algebra with central charges? To answer this question one has to use the fact that

$$
\left(P^{A} \gamma_{A} C\right)\left(P^{B} \gamma_{B} C\right)^{\dagger}=P^{A} P_{A} \mathrm{I}
$$

where $\mathrm{I}$ is a unit matrix. To get the same diagonal structure from $\left(C_{4}\right)_{\alpha \beta} U^{i j}+\left(\gamma_{5}\left(C_{4}\right)\right)_{\alpha \beta} V^{i j}$ one has to require that the matrix of the central charges in the form in eq. (5), to which it can be brought after appropriate change of the basis, has to be proportional to the unit matrix

$$
\left(\begin{array}{cc}
z_{1} & 0 \\
0 & z_{2}
\end{array}\right)=\left(P_{A} P^{A}\right)^{\frac{1}{2}}\left(\begin{array}{cc}
1 & 0 \\
0 & 1
\end{array}\right)
$$

which requires $z_{1}=z_{2}$. When the four-dimensional mass $m_{4}$ is equal to the value of the central charges, one has

$$
m_{4}^{2} \equiv\left(-P_{\mu} P^{\mu}\right)^{\frac{1}{2}}=\left(P_{A} P^{A}\right)^{\frac{1}{2}}, \quad m_{10}^{2} \equiv\left(-P_{\mu} P^{\mu}\right)^{\frac{1}{2}}-\left(P_{A} P^{A}\right)^{\frac{1}{2}}=0
$$

In conclusion, the centron supermultiplet satisfying a Bogomolny bound of $N=4$ supersymmetry with the highest spin state 1 in four dimensions, can be reinterpreted as the light-like representation of $d=10, N=1$ global SUSY. The deep relation between supersymmetric gravitational pp-waves and uplifted into ten dimensions extreme black holes [9] holes may be related to this property of the supersymmetric multiplets.

Thus we have also learned that centrino multiplet of the four-dimensional SUSY, which extends the range of a spin up to $\frac{3}{2}$, does not form any representation of the ten-dimensional global SUSY. Indeed, the mere existence of the centrino is based on the central charges split (8), (11). Ten-dimensional global SUSY forbids such split, according to eq. (20). However, centrino is a perfect representation of $d=4, N=4$ supersymmetry.

We would like to use the existence of massive soliton supermultiplets in extended supersymmetries for the purpose of spontaneous breaking of local supersymmetry. In what follows we will discuss one possible way of thinking about it.

\section{$5 \quad$ Gluino condensation induced by Killing spinors}

All known Lagrangian theories of local $d=4, N=4$ supersymmetry describe only massless supermultiplets. However, supersymmetric gravitational solitons (solutions of the full non-linear field equations) do bring into the theory mass parameters via the ADM mass. Some of these configurations which admit supercovariantly constant spinors form the massive supermultiplets discussed above.

The purpose of this section is to show that black holes with unbroken supersymmetries may be capable of affecting the massless sector of $N=4$ theory by inducing a gluino condensate without a cosmological constant and therefore (at least some of those solutions) may supply 
their Killing spinors for consistent spontaneous symmetry breaking. We will present here the observation about all configurations with unbroken $N=4$ supersymmetry which may be useful for the investigation of their possible role in particle physics in connection with spontaneous breaking of local supersymmetry.

Suppose that extreme black holes (or their instanton counterparts which appear after uplifting black holes to $\mathrm{d}=10$ and then reducing them back to Euclidean $\mathrm{d}=4$ space) may induce the gluino condensate. One can then address the following question: if some dynamical mechanism exists for such condensation, what are the properties of the condensate in this theory?

The fundamental property of the Killing spinors admitted by extreme black holes in asymptotically flat space-times is the fact that they do not fall off at infinity. Near the horizon they behave differently, depending on the configuration and on whether one works in canonical or stringy frame. In particular, some of extreme black holes have Killing spinors vanishing at the horizon in canonical four-dimensional geometry, some of them have Killing spinors remaining constant in the stringy frame. Some extreme black holes in canonical four-dimensional geometry have the singularity hidden by the horizon, some have singular horizons, and some exhibit naked singularities. However, those with singular horizons and/or naked singularities have a vanishing area of the horizon. Because of these various unusual properties there is not much information available at the moment about the normalizable fermion zero modes in the extreme black hole backgrounds. The systematic study of such fermion modes would be most desirable and would clarify the issues which are raised in this paper.

Most of the solutions with unbroken $N=4$ supersymmetries in four dimensions has been uplifted to ten dimensions and most of the $N=1$ ten-dimensional supersymmetric solutions have been dimensionally reduced down to four. The way up and down is not completely unique, there are duality transformations on the way. Taking into account this ambiguity one may still have a clear identification of solutions in ten and four dimensions depending on whether they saturate a single or a double supersymmetric bound, i.e. whether they have one half or one quarter of unbroken supersymmetries.

We have found that Killing spinors of ten-dimensional supergravity configurations automatically solve the gluino field equations in the background given by the corresponding configuration. To establish that, one may consider, e. g., the following combination of equations for the Killing spinors of ten-dimensional supergravity:[1]

$$
\gamma^{a} \delta \psi_{a}+2 \sqrt{2} \delta \lambda=0, \quad a=0,1, \ldots, 9 .
$$

Using the explicit expression for the variation of the ten-dimensional gravitino

$$
\delta \psi_{a}=D_{a+} \epsilon \equiv\left[D_{a}(\omega)-\frac{3 \sqrt{2}}{8} H_{a b c} \gamma^{b c}\right] \epsilon(x)
$$

and dilatino

$$
\delta \lambda=\left[-\frac{3 \sqrt{2}}{8} \phi^{-1} \gamma^{a} \partial_{a} \phi+\frac{1}{8} H_{a b c} \gamma^{a b c}\right] \epsilon(x)
$$

\footnotetext{
${ }^{7} \mathrm{We}$ are using the description of ten-dimensional theory in [10]. For the time being we consider only the classical supergravity coupled to Yang-Mills theory.
} 
(with all fermions vanishing) one can verify that the combination above is proportional to the part of equation of motion for the gluino in the external field of the uplifted black hole, which is linear in the gluino,

$$
\left[\gamma^{a} D_{a}(\omega)-\frac{3}{2} \phi^{-1} \gamma^{a} \partial_{a} \phi-\frac{\sqrt{2}}{8} \gamma^{a b c} H_{a b c}\right] \chi(x)=0 .
$$

The part of the gluino equation coming from the the 4-gluino term is proportional to

$$
\gamma^{a b c} \chi \operatorname{tr}\left(\bar{\chi} \gamma_{a b c} \chi\right) \text {. }
$$

This term will vanish either when gluino belong to an Abelian group or as a consequence of some constraints. For example, if the non-Abelian gluino satisfies the light-cone constraint, which we will discuss later, this term will drop from the equations of motion for gluino. One also has to take into account that in the presence of gluino the supersymmetry variation of the gravitino, as well as of the dilatino, is modified according to [10],

$$
\begin{aligned}
\delta_{\beta} \psi_{a} & =\frac{1}{192} \beta \gamma^{b c d} \Gamma_{a} \epsilon(x) \operatorname{tr}\left(\bar{\chi} \gamma_{b c d} \chi\right), \\
\delta_{\beta} \lambda & =\frac{1}{384} \beta \gamma^{b c d} \epsilon(x) \operatorname{tr}\left(\bar{\chi} \gamma_{b c d} \chi\right),
\end{aligned}
$$

where $\beta$ is a constant in front of the Yang-Mills multiplet.

We would like to find a solution of the gluino field equations using the Killing spinors of the configuration without fermions first. Note that the gluino has the same ten-dimensional chirality as the parameter of the supersymmetry transformations. Afterwards we may investigate whether we still have Killing spinors in the presence of the non-vanishing gluino. We may further look for the gluino condensate using the Killing spinors to construct the bilinear combination of gluinos. We will study various possibilities for different type of configurations admitting Killing spinors. Finally the effect of the gluino condensate on the local supersymmetry transformations of the gravitino and the dilatino can be studied.

The main feature of any uplifted extreme black hole configuration is the number and the form of the linear constraints which the Killing spinors satisfy. Besides, the uplifted black holes depend only on $x^{1}, x^{2}, x^{3}$. Therefore they form a subset of all configurations which have unbroken supersymmetries in ten dimensions. For simplicity we will call them uplifted black holes with the understanding that as the ten-dimensional configuration some of them may look as pp-fronted gravitational waves or instantons of the four-dimensional submanifold or monopoles.

The large set of ten-dimensional supersymmetric configurations includes supersymmetric waves, fundamental strings, dual waves, chiral null models, etc. They may also depend on $x^{4}, x^{5}, x^{6}, x^{7}, x^{8}$, but typically they are independent of $x^{0}$ and $x^{9}$. Some of them are related to uplifted electrically charged black holes. The corresponding Majorana-Weyl ten-dimensional Killing spinor satisfies the light-cone condition

$$
\gamma^{+} K \equiv\left(\gamma^{0}+\gamma^{9}\right) K=0 .
$$


Magnetically charged uplifted black holes usually are constrained by the chirality condition

$$
\left(1+\gamma^{5}\right) K \equiv\left(1+\gamma^{1} \gamma^{2} \gamma^{3} \gamma^{4}\right) K=0 .
$$

In both cases the number of Killing spinors is equal to 8 since both the light-cone constraint (29) as well as the chiral constraint (30) break any spinor into two parts and the unconstrained spinor is 16-dimensional. Pure electric configurations can be brought to pure magnetic ones by the $S L(2, R)$ rotation. In ten-dimensional geometry this corresponds to a rotation of the Killing spinor from the light-cone one to the chiral one. Both are eight-dimensional.

Uplifted $U(1)^{2}$ black holes with one quarter of unbroken supersymmetry satisfy both the lightcone as well as chirality constraints. [T The first condition leaves one half of the 16-component ten-dimensional spinor, the second condition leaves one half of the remaining 8 components. Thus in any case we get a 4-component Killing spinor. In the limit that the magnetic charge vanishes, we get only the first light-cone constraint and the Killing spinor becomes an 8-component light-cone spinor, like the Killing spinor of the ten-dimensional pp-fronted supersymmetric waves. When the electric charge vanishes, we get an 8-component Killing spinor which is chiral in 4-dimensional Euclidean manifold $x^{1}, x^{2}, x^{3}, x^{4}$. This is a standard property of Killing spinors of the monopoles, instantons, and uplifted magnetic black holes. However, as long as both charges of two different $U(1)$ fields are present in the solution, which provides the split of central charges, the Killing spinor has to satisfy both constraints, given in eqs. (29), (30). The torsionful curvature of the ten-dimensional space has both some null properties in $u=t-9$ direction and is self-dual in the four-dimensional Euclidean space 1,2,3,4.

Having established the constraints (29), (30), which are satisfied by Killing spinors, we may proceed with the investigation of the 3-gluino term (26). The linear part of gluino field equations is solved by Killing spinors for any non-Abelian gluino. Therefore in the first approximation we can take any of the components the non-Abelian gluino field $\chi$ to be proportional to a Killing spinor $K$. However, if we would like also to get rid of the 3-gluino term (26) we may either rely on the light-cone constraint for gluino, which makes the 3-gluino term vanishing, or just choose only an Abelian gluino field. The 3-gluino term vanishes for any Abelian ten-dimensional Majorana-Weyl spinor. This second possibility also makes the corrections to the Killing spinor equations due to gluino vanishing. Having all these options in mind we may look for the gluino condensate using the Killing spinors to construct the bilinear combination of gluinos.

All black holes which admit Killing spinors have horizons, when considered in canonical $d=4$ geometry. In general, we are not aware of supersymmetric solitons in the theory of gravity in asymptotically flat four-dimensional space which are free of horizons and singularities. Rather, one may divide all known solutions as to whether the horizon is singular or whether it is not and covers the singularity hidden behind the horizon. The first type of the solutions seems mostly related to the states saturating the strong Bogomolny bound (double bound of $N=4$

\footnotetext{
${ }^{8}$ Our four-dimensional choice of electric and magnetic $U(1)$ directions was $\alpha^{3}$ for the vector field and $\beta^{3}$ for the axial-vector field [1]. This corresponds to $x^{6}$ for the electric field and $x^{9}$ for the magnetic one. We prefer to use as an electric direction the $x^{9}$-coordinate and as the magnetic one the $x^{4}$-coordinate. With such a choice the meaning of the one quarter of unbroken supersymmetry becomes very clear.
} 
supersymmetry). In particular, pure electric or pure magnetic $a=1$ or $a=\sqrt{3}$ dilaton black holes have singular horizon with vanishing area in canonical geometry. All such solutions have one half of unbroken $N=4$ supersymmetry, i.e. the Killing spinor has 8 components.

On the other hand, known $U(1)^{2}$ black holes [1] have 4 Killing spinors which tend to a constant far away from the black hole and vanish at the horizon. In addition, these black holes have regular horizon in canonical four-dimensional geometry. The singularity of these extreme black holes is behind the horizon as long as the central charges are split, $z_{1}>z_{2}$, since the singularity is at $r=z_{2}$ whereas the event horizon is at $r_{+}=m=z_{1}>z_{2}$. These solutions saturate only a single bound of $N=4$ supersymmetry.

As a simplest possibility we may take an uplifted Reissner-Nordström dyon with equal electric and magnetic charges, $m=z_{1}, z_{2}=0$. This particular solution has no dilaton, the stringy frame coincides with the canonical one in four as well as in ten dimensions. From the uplifted anticommuting covariantly constant Killing spinors $K=\sqrt{1-\frac{m}{r}} \epsilon_{0}$ satisfying the constraints (29), (30) one can construct their quadratic combination which may serve as a possible form of gluino condensate as follows:

$$
\aleph^{a b c} \equiv \operatorname{tr}\left(\bar{\chi} \gamma^{a b c} \chi\right)=C \bar{K} \gamma^{a b c} K=C\left(1-\frac{m}{r}\right) \bar{\epsilon}_{0} \gamma^{a b c} \epsilon_{0}, \quad a=0,1, \ldots 9
$$

Here $\gamma^{a b c}$ is a completely antisymmetrized product of three gamma matrices in the tangent space, $\epsilon_{0}$ is a constant spinor, satisfying both constraints (29), (30). The dependence of the condensate on the distance from the black hole is $\left(1-\frac{m}{r}\right), r^{2}=(\vec{x})^{2}=\left(x^{1}\right)^{2}+\left(x^{2}\right)^{2}+\left(x^{3}\right)^{2}$; it comes from the dependence of the Killing spinors on the geometry, . The constant $C$ is introduced to take care of the dimension of the gluino versus the Killing spinor.

In isotropic coordinates which at positive $\rho=r-m$ describe only the space outside the horizon we have

$$
\aleph^{a b c}=C\left(1+\frac{m}{\rho}\right)^{-1} \bar{\epsilon}_{0} \gamma^{a b c} \epsilon_{0}, \quad a=0,1, \ldots 9 .
$$

When $\rho \rightarrow \infty$ the condensate tends to a constant, near the horizon when $\rho \rightarrow 0$ the condensate vanishes. For the multi black hole solutions we get

$$
\aleph^{a b c}=C\left(1+\Sigma_{s} \frac{m_{s}}{\left|\vec{x}-\vec{x}_{s}\right|}\right)^{-1} \bar{\epsilon}_{0} \gamma^{a b c} \epsilon_{0}, \quad a=0,1, \ldots 9,
$$

where $\vec{x}_{s}, s=1, \ldots, n$ is the position of each black hole.

The non-vanishing components of $\aleph^{a b c}$ can be chosen to be

$$
\aleph^{-s t} \equiv \operatorname{tr}\left(\bar{\chi} \gamma^{-s t} \chi\right), \quad \gamma^{-}=\gamma^{0}-\gamma^{9}, \quad s, t=5,6,7,8 .
$$

The reason for this choice is the following. To satisfy the light-cone constraint (29) we have to choose at least one out of $\gamma^{a}, \gamma^{b}, \gamma^{c}$ to be $\gamma^{-}$and the rest could be in any part of $S O(8), a=$

\footnotetext{
9 It would be very interesting to find solutions, saturating the double bound with non-singular horizon in canonical four-dimensional geometry.
} 
$1, \ldots, 8$. For the rest we take into account that we have only a four-component anticommuting Killing spinor, therefore there exists a six-component bilinear combination of such spinors. Since we are interested in a combination whose vacuum expectation value does not vanish, we choose out of all possible $S O(8)$ antisymmetric tensors only the part of it belonging to $S O(4)$ since directions $5,6,7,8$ are flat. The equivalent statement about the condensate is the following. There exists a covariantly constant bilinear combination of the covariantly constant Killing spinors

$$
\aleph_{[\alpha \beta]} \equiv C K_{\alpha} K_{\beta}, \quad D_{\mu+}\left(\aleph_{[\alpha \beta]}\right)=0,
$$

which provides the gluino condensate. This is a natural choice for the Reissner-Nordström dyon with one quarter of unbroken supersymmetries.

This choice is acceptable also for all solutions with light-cone Killing spinor with one half of unbroken supersymmetries. The square of the gluino condensate, which is build out of light-cone anticommuting Killing spinors, vanishes since the condensate has a null property. Namely, since the component $\aleph^{+s t}$ vanishes due to the light-cone constraint (29) we have

$$
\left(\aleph^{a b c}\right)^{2} \equiv\left(\operatorname{tr}\left(\bar{\chi} \gamma^{a b c} \chi\right)\right)^{2}=\operatorname{tr}\left(\bar{\chi} \gamma^{-s t} \chi\right) \operatorname{tr}\left(\bar{\chi} \gamma_{-s t} \chi\right)=0 .
$$

The contribution to the gluino field equation from the 4-gluino term also vanishes, since

$$
\operatorname{tr}\left(\bar{\chi} \gamma^{-s t} \chi\right) \gamma_{-s t} \chi=0 .
$$

For magnetic solutions one can also use this choice, by choosing only those of 8 Killing spinors which satisfy the light-cone constraint.

Thus the gluino condensate may appear in the supersymmetry transformation of the gravitino and dilatino and may lead to spontaneous violation of supersymmetry. Also it may induce a mass for the gravitino via the 2-gravitino-2-gluino-interaction terms. There will be no cosmological constant ${ }^{0}$ due to the condition (36). A possible scenario is the following. One may start with the action of the ten-dimensional supergravity coupled to Yang-Mills fields. Since the Killing spinor, which does not vanish at infinity, solves the equations of motion of gluino, we may shift the gluino field on the Killing spinor.

$$
\chi \rightarrow \chi+C K .
$$

The new gluino will be a well behaved field and appropriate for the standard formulation of the quantum field theory (i.e. it will have the behavior at infinity which is appropriate for a field over which one integrates in the path integral). This reminds us of the situation with the separating the non-vanishing at infinity vacuum expectation of the Higgs field. After this procedure we will get in the local supersymmetry transformation rules for gravitino and dilatino all kind of usual terms, depending on fields of the theory which fall off at infinity and vanish near the horizon.

\footnotetext{
${ }^{10}$ The mechanism of gluino condensation studied in [3] was developed for the Calabi-Yau manifold. It was necessary there to compensate the gluino condensate by a 3 -form condensate, since there was only one non-null possible structure related to an $S U(3)$ holonomy of the six manifold.
} 
The exceptional terms will be

$$
\begin{aligned}
\delta \psi_{a} & =\ldots+\frac{1}{192} \beta \gamma^{b c d} \Gamma_{a} \epsilon(x) C\left(1+\frac{m}{\rho}\right)^{-1} \operatorname{tr}\left(\bar{\epsilon}_{0} \gamma_{b c d} \epsilon_{0}\right), \\
\delta \lambda & =\ldots+\frac{1}{384} \beta \gamma^{b c d} \epsilon(x) C\left(1+\frac{m}{\rho}\right)^{-1} \operatorname{tr}\left(\bar{\epsilon}_{0} \gamma_{b c d} \epsilon_{0}\right) .
\end{aligned}
$$

Here $\epsilon(x)$ are generic local supersymmetry parameters depending on ten-dimensional coordinates, $\epsilon_{0}$ are the constant spinors representing the Killing spinors far away from the black hole.

It would be interesting to relate this observation to the properties of the moduli space of the system of two black holes. It was found in [11] that the Euler number for the Reissner-Nordström case equals 1, which may be a signal of spontaneous breaking of supersymmetry. Indeed, if one interprets the Euler number of the moduli space manifold as the Witten index, one discovers that the supersymmetry is broken. For a two-black hole system with $a^{2}=1$ the moduli space has a conical singularity, and for $a^{2}=3$ the moduli space is flat.

In conclusion, we suggest to use the centrino as well as the centron multiplets to break local supersymmetry, for example by inducing gluino condensation. From the point of view of the tendimensional stringy geometry magnetic type centrons with the first component of the multiplet given by the magnetically charged black holes are most attractive: stringy $\alpha^{\prime}$-corrections can be taken care of by embedding the spin connection into the gauge group. Electric centrons also have advantages since at least some of them belong to a family of supersymmetrized pp-fronted ten-dimensional gravitational waves and also permit the embedding of the spin connection into the gauge group. Note that magnetic and electric charges of these configurations are the central charges of the supersymmetry algebra.

From the point of view of a four-dimensional canonical geometry, centrino multiplets related to Reissner-Nordtsröm-type $U(1)^{2}$ dyon black holes [1] seem to deserve special attention. Indeed, they contain a massive spin $\frac{3}{2}$ state (without a massive spin 2 state). It would be most interesting to build the effective action for this multiplet and investigate the possibilities to mix it with gravitino. The fact that the natural structures for the gluino condensation are provided by the centrino black hole looks encouraging. However, much more work will be required to understand whether indeed all consistency requirements for the super-Higgs mechanism can be met. The scenario of spontaneous breaking of local supersymmetry via massive supersymmetric configurations with central charges outlined above needs to be supported by a specific dynamical mechanism.

I am very grateful to R. Brooks, A. Linde, T. Ortín, S.-J. Rey and L. Susskind for many fruitful discussions of the issues studied in this paper.

\section{References}


[1] R. Kallosh, A. Linde, T. Ortín, A. Peet, and A. Van Proeyen, Phys. Rev. D 46, 5278 (1992).

[2] J. Strathdee, Int. J. Mod. Phys. A2, 273 (1987).

[3] M. Dine, R. Rohm, N. Seiberg and E. Witten, Phys. Lett. 156B, 55 (1985).

[4] E. Witten and D. Olive, Phys. Lett. 78B, 97 (1978).

[5] G.W. Gibbons, in: Lecture Notes in Physics 160, Proceedings of the Heisenberg Memorial Symposium 1981, eds. P.Breitenlohner and H. P. Dürr, (Springer-Verlag 1982);

G.W. Gibbons and C.M. Hull, Phys. Lett. 109B, 190 (1982).

[6] S. Ferrara, C. A. Savoy and B. Zumino, Phys. Lett. 100B, 393 (1981).

[7] J. H. Schwarz and A. Sen, Phys. Lett. B312, 105 (1993);

A. Sen, Int. J. Mod. Phys. A9, 3707 (1994).

[8] M. J. Duff and J. Rahmfeld, "Massive string states as extreme black holes," preprint CTPTAMU-25/94, hep-th/9406105.

[9] E. Bergshoeff, R. Kallosh, T. Ortín, Phys. Rev. D 50, 5188-5192 (1994);

G. T. Horowitz and A. A. Tseytlin, "A new class of exact solutions in string theory," preprint Imperial/TP/93-94/54, UCBTH-94-31, hep-th/9409021 (1994);

K. Behrndt, "The 10-D chiral null model and the relation to 4-D string solutions," preprint DESY 94-237, hep-th/9412162 (1994).

[10] E. Bergshoeff and M. de Roo, Nucl. Phys. B328, 439 (1989); Phys. Lett. B218, 210 (1989).

[11] G. W. Gibbons and R. E. Kallosh, "Topology, Entropy and Witten Index of Dilaton Black Holes," preprint NI94003, hep-th/9407118, to be published in Phys. Rev. D. 